\title{
Migration und Entwicklung : Standpunkt der Politischen Abteilung IV (EDA)
}

Pietro Mona

\section{(2) OpenEdition \\ 12 Journals}

Electronic version

URL: http://journals.openedition.org/sjep/342

DOI: 10.4000/sjep.342

ISSN: 1663-9677

Publisher

Institut de hautes études internationales et du développement

Printed version

Date of publication: 1 décembre 2008

Number of pages: 197-206

ISBN: 978-2-940415-08-3

ISSN: $1660-5926$

\section{Electronic reference}

Pietro Mona, « Migration und Entwicklung : Standpunkt der Politischen Abteilung IV (EDA) », doi.org/10.4000/sjep.342 


\title{
Migration und Entwicklung: Standpunkt der Politischen Abteilung IV (EDA)
}

\author{
Pietro Mona*
}

\begin{abstract}
„Die Geschichte der Menschheit ist immer auch als Wanderungsgeschichte zu verstehen. Migration hat den Prozess des globalen Wirtschaftswachstums unterstützt, zur Entwicklung der Staaten und Gesellschaften beigetragen und viele Kulturen bereichert. Migranten gehören häufig zu den dynamischsten und unternehmerischsten Mitgliedern einer Gesellschaft. Sie sind Menschen, die bereit sind, sich über die Grenzen ihrer eigenen Gemeinschaft und ihres eigenen Landes zu wagen, um sich selbst und ihren Kindern neue Perspektiven zu eröffnen. ${ }^{~}$
\end{abstract}

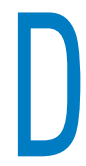
as obige Zitat aus dem Bericht der Weltkommission für Internationale Migration (GCIM) zeigt zentrale Aspekte des Nexus zwischen Migration und Entwicklung auf. Obwohl diesem synergetischen Zusammenspiel erst seit einigen Jahren eine besondere Aufmerksamkeit geschenkt wird, hat die Migration seit jeher die menschliche und gesellschaftliche Entwicklung mitgeprägt. Man könnte geneigt sein, den Entwicklungsbeitrag der Migration, sei es im Herkunfts- oder im Zielland, mit einem genauen Betrag beziffern zu wollen. Migranten $^{2}$ sind jedoch weit mehr als blosse Arbeitskräfte, deren volkswirtschaftlichen Nutzen man errechnen könnte. Sie bereichern die Staaten und die Gesellschaft auch hinsichtlich des sozialen und kulturellen Lebens. Man sollte sie daher als Agenten einer breit gefächerten und dynamischen Entwicklung betrachten, deren Potenzial man nunmehr auch im nationalen und internationalen Rahmen explizit wahrzunehmen beginnt und spezifisch zu fördern versucht.

Wir stehen sicherlich noch am Anfang eines langen Weges voller unausgeschöpfter Möglichkeiten. Die Synergiebildung zwischen Migration und Entwicklung bedeutet allerdings nicht die Lösung aller Entwicklungsprobleme dieser Welt. Umgekehrt wird die Entwicklungszusammenarbeit auch nicht zur vollständigen Unterbindung irregulärer Migrationsbewegungen führen. Entwicklungszusammenarbeit trägt aber dazu bei, die Lebenssituation der Ärmsten

* Politischer Berater, Sektion humanitäre Politik und Migration, Politische Abteilung IV Menschliche Sicherheit (PA IV), Eidgenössisches Departement für auswärtige Angelegenheiten (EDA).

Anmerkung: Der Beitrag wurde im Frühjahr 2008 geschrieben. Gewisse Angaben zur schweizerischen Politikentwicklung im Bereich Migration und Entwicklung oder betreffend regionale und internationale Konferenzen entsprechen daher möglicherweise nicht mehr dem Stand der Dinge zum Zeitpunkt der Publikation.

1 Global Commission on International Migration (GCIM), Migration in an Interconnected World - New Directions for Actions, 2005, <http://www.gcim.org>.

2 Der einfacheren Lesbarkeit halber wird in diesem Beitrag jeweils nur die männliche Form verwendet, obwohl damit jeweils ,Migranten und Migrantinnen“ gemeint sind. 
zu verbessern, was einer erzwungenen Migration aus Not und Elend durchaus vorbeugen kann.

Den Staaten obliegt es, das Potenzial einer engeren konzeptionellen und operationellen Verknüpfung migrations- und entwicklungspolitischer Instrumente zu fördern und zu nutzen. Die Schweiz versucht dieser Herausforderung sowohl intern, durch eine engere interdepartementale Zusammenarbeit im Sinne eines ganzheitlichen Ansatzes (,whole-of-government approach“), als auch im regionalen und internationalen Rahmen, durch die aktive Teilnahme an ausgewählten Initiativen wie dem UN High-level Dialogue on Migration and Development oder dem Globalen Forum für Migration und Entwicklung (Global Forum on Migration and Development), gerecht zu werden. Das Eidgenössische Departement für auswärtige Angelegenheiten (EDA) nimmt hierbei in seiner Funktion als Scharnierstelle zwischen In- und Ausland eine besondere Rolle ein, indem es die migrationspolitischen Interessen der Schweiz im Ausland vertritt und gleichzeitig die aussenpolitische Perspektive in den internen Diskurs mit einfliessen lässt.

\section{Migration und Entwicklung im Sinne eines "whole-of-government approach"}

Der ganzheitliche Regierungsansatz (,whole-of-government approach“) ist zum Leitmotiv nationaler Bestrebungen geworden, das komplexe und vielschichtige Thema der Migration durch eine kohärente und zielorientierte Politik aller relevanten Regierungsstellen anzugehen. Über die Jahre hat man mehr und mehr festgestellt, dass eine nationale Migrationspolitik, die losgelöst von anderen Politikbereichen betrieben wird, der Komplexität dieses Themengebiets nicht ausreichend Rechung tragen kann. In diesem Sinne erhielt die interdepartementale Arbeitsgruppe „Aussenpolitik im Migrations- und Rückkehrbereich“ (IDAG Migration) 2003 vom Bundesrat folgenden Auftrag: „Die IDAG Migration hat Mittel und Wege diskutiert, um die aussenpolitischen Instrumente für eine migrationspolitische Gesamtstrategie nutzbar zu machen. Sie suchte weiter nach möglichen Synergien zwischen den verschiedenen aussenwirksamen Aktivitäten der Departemente und Bundesämter im Bereich Migration und Rückkehr sowie nach möglichen institutionellen Verbesserungen der interdepartementalen Koordination im Migrationsbereich. " ${ }^{3}$

Eine kohärente Migrationspolitik muss die Aussen- und Innenperspektive verbinden und die lokalen Behörden, den Privatsektor, Einrichtungen der Zivilgesellschaft, akademische Institutionen und Migrantenverbände bei ihrer Formulierung und Umsetzung einbinden. Sie bringt keine schnellen Lösungen, weil Interessenkonflikte im gegenseitigen Dialog sorgfältig abgewogen und einer gemeinsamen Lösung zugeführt werden sollen. Sie überwindet jedoch sektorielles Denken und Handeln, das im Widerspruch zum Facettenreichtum des Migrationsphänomens steht.

Dieser Ansatz wird auch deshalb verfolgt, weil der Dialog mit den Herkunftsund Transitstaaten die Bundesbehörden vor neue Herausforderungen stellt. Zunehmend machen diese Staaten im Gegenzug zur Rückübernahme ihrer

3 IDAG Migration, Schlussbericht der IDAG Migration, Bern, 2004, <http://www.eda.admin.ch/etc/ medialib/downloads/edazen/topics/migr.Par.0001.File.tmp/Schlussbericht_Interdept_AG_Migration_ de.pdf>. 
Staatsangehörigen eigene Interessen geltend. Einen Interessenausgleich können die mit dem Vollzug beauftragten Behörden nicht allein leisten, denn die vorgebrachten Anliegen der Herkunfts- und Transitstaaten betreffen oft Politikbereiche (wie zum Beispiel die Entwicklungspolitik), die nicht in ihrer Kompetenz liegen. Daraus ergibt sich ein steigender Koordinations- und Kooperationsbedarf, dem durch interdepartementale Gremien entsprochen werden muss.

Synergien lassen sich, ganz im Sinne des bereits erwähnten Berichts der Arbeitsgruppe IDAG Migration, auch in weiteren aussenpolitischen Aktionsfeldern der Schweiz herbeiführen. Man denke zum Beispiel an die Aktivitäten im Bereich der zivilen Friedensförderung, der Förderung der Menschenrechte oder der Stärkung des Schutzes der Zivilbevölkerung. Die Relevanz dieser Aktionsfelder in Bezug auf die Migration wurde unter anderem auch in der parlamentarischen Debatte über die Weiterführung der Massnahmen zur zivilen Friedensförderung und Stärkung der Menschenrechte mehrfach hervorgehoben ${ }^{4}$. Aufgrund des thematischen Schwerpunktes der vorliegenden Publikation soll jedoch primär der Nexus zwischen Migration und Entwicklung hervorgehoben werden.

Seit über zehn Jahren formuliert die Schweiz verschiedene Bereiche ihrer Migrationspolitik im interdepartementalen Dialog. Diese Zusammenarbeit bewährte sich zunächst im Rahmen der Programme zur Rückkehr auf den Balkan, als die Interdepartementale Leitungsgruppe Rückkehrhilfe (ILR) gegründet wurde. Mittlerweile setzt die Schweiz in mehreren anderen Ländern (etwa Nigeria, Äthiopien, Irak und Afghanistan) erfolgreich Rückkehrhilfeprogramme um. Seit 1997 koordiniert die ILR so die Rückkehr von Tausenden von Menschen in ihre Herkunftsländer. Einerseits geht es dabei um individuelle Rückkehrhilfe, andererseits finanziert das Bundesamt für Migration auch Strukturhilfeprojekte, die von der Direktion für Entwicklung und Zusammenarbeit (DEZA) vor Ort umgesetzt werden. In vielen Regionen verbindet die Schweiz auf diese Weise seit Jahren operationelle Migrationspolitik und Entwicklungspolitik im Rahmen eines ,whole-of-government approach“ mit grossem Erfolg.

Augrund des Berichts der IDAG Migration wurde zudem der Ausschuss „Focus Migration“ der Interdepartementalen Arbeitsgruppe Migration (IAM) gegründet. Der Ausschuss dient dazu, die Interessen der einzelnen Bundesstellen hinsichtlich der migrationsbezogenen Herausforderungen grundsätzlich zu diskutieren, eine gemeinsame Strategie festzulegen und die zur Verfügung stehenden innen- und aussenpolitischen Instrumente kohärent und konsequent in migrationspolitisch relevanten Ländern anzuwenden. Zudem sollen, gestützt auf Artikel 100 des Ausländergesetzes, Migrationspartnerschaften mit punktuell ausgewählten Staaten angestrebt werden.

Bei Migrationspartnerschaften sollen die unterschiedlichen Interessen der beteiligten Länder berücksichtigt werden. Im Entwicklungsbereich etwa schlagen sie Brücken zwischen den Bedürfnissen der nördlichen, südlichen und östlichen Länder. Sie schaffen eine Verbindung zwischen der Rückkehrproblematik und den Ursachen von erzwungener Migration (u.a. Armut, Konflikte und Menschenrechtsverletzungen). Eine Migrationspartnerschaft schafft durch den gegenseitigen Austausch Verständnis für die Positionen der anderen und eröffnet

4 Siehe amtliches Bulletin, Wortprotokolle von Nationalrat und Ständerat unter: <http://www.parlament. ch/ab/frameset/d/n/4802/263473/d_n_4802_263473_263474.htm>. 
so neue Wege für konstruktive Lösungen zur Bewältigung der globalen Migrationsproblematik.

Kohärenz, Koordination und Kooperation - die so genannten „drei K’s“ - sind nicht nur notwendig, um eine zielorientierte nationale Migrationspolitik zu führen, sondern sie bilden auch die Grundlage für eine effiziente regionale und internationale Zusammenarbeit im Migrationsbereich.

\section{Internationaler Dialog zum Thema „Migration und Entwicklung“}

Der internationale Dialog über Migration und Entwicklung kann nicht auf einzelne Veranstaltungen oder Prozesse reduziert werden. Vielmehr ist es die Summe aller globalen, regionalen und subregionalen Initiativen und Prozesse, die zur Gesamtdiskussion beitragen. Einige Migrationsthemen sind globaler Natur und sollten daher auf globaler Ebene diskutiert werden. Für andere Themen scheinen regionale und subregionale Foren angemessener, um massgeschneiderte Resultate zu definieren und umzusetzen. Die verschiedenen Debatten sollten aber ineinander übergehen und sich gegenseitig bereichern.

\section{Der Rabat Prozess: Beispiel einer regionalen Initiative}

Das Konzept der Migrationspartnerschaften, das von der Idee geleitet wird, gemeinsam die negativen Aspekte der Migration zu bekämpfen und die positiven Aspekte zu fördern und zu nutzen, stützt sich auf die Bereitschaft zum Dialog und zur Zusammenarbeit. Dieselbe Überlegung ist auch Leitmotiv des sog. Rabat-Prozesses, der im Juli 2006 lanciert wurde.

Zum ersten Mal diskutierten in Rabat (Marokko) Ursprungs-, Transit- und Zielstaaten aus Zentral-, West und Nordafrika sowie aus Europa gemeinsam den Nexus zwischen Migration und Entwicklung. Schwerpunkte der Diskussionen waren einerseits Formen der legalen Migration und die Bekämpfung der irregulären Formen der Migration - insbesondere Menschenhandel und Menschenschmuggel -, andererseits die vielfältigen Zusammenhänge zwischen Entwicklungspolitik und Migration.

Der Massenansturm auf die spanischen Exklaven Ceuta und Melilla, die Nachrichten von der italienischen Insel Lampedusa und aus Malta sowie die steigende Zahl gestrandeter Migranten auf den Kanarischen Inseln hatten die Staatengemeinschaft aufgerüttelt und dazu bewogen, diesem regionalen Phänomen durch einen partnerschaftlichen und regionalen Ansatz neu zu begegnen. Gleichzeitig war man sich bewusst, dass die Partnerschaft zwischen Süd und Nord auch durch unterschiedliche Interessen gekennzeichnet ist. Auf einem Kontinent, wo rund die Hälfte der Bevölkerung jünger als 20 Jahre ist, wo bereits heute dreimal mehr junge Arbeitsuchende auf den Arbeitsmarkt strömen, als Arbeitsplätze zur Verfügung stehen, und wo viele Staaten von Korruption und Menschenrechtsverletzungen geprägt sind, wächst der Drang, in den Norden zu wandern, unweigerlich. Den dadurch steigenden Migrationsdruck kann und will man in seiner bisherigen Form in den nördlichen Staaten nicht auffangen. Die Lösung dieser Problematik sucht man deshalb in einer pragmatischen und ausgeglichenen Partnerschaft zwischen den betroffenen Staaten. Die Kon- 
trolle der Migrationsflüsse soll sich nicht mehr einzig auf die territoriale Grenzüberwachung beschränken, sondern soll vermehrt auch den Zugang zu legalen Migrationsmöglichkeiten sowie erhöhte Anstrengungen zur Entwicklung des afrikanischen Kontinents beinhalten.

Regionale und subregionale Institutionen und Prozesse bleiben der von vielen Staaten bevorzugte Rahmen für eine Zusammenarbeit. In der Tat sind solche Instrumente geeignet, um ein gemeinsames Verständnis zu entwickeln und spezifische Aspekte der Migrationspolitik zu vertiefen. Es ist wichtig, dass ein solcher Dialog im Sinne einer Partnerschaft geführt wird. Der von der Schweiz angestrebte Ansatz der bilateralen Migrationspartnerschaften soll daher unser Handeln auch bei solchen Prozessen lenken.

\section{Globale Perspektive}

Migration wird immer stärker auch durch globale Migrationsbewegungen gekennzeichnet: Ursprünglich aus Asien stammende Migranten versuchen in stetig wachsender Zahl, auch über den afrikanischen Kontinent nach Europa zu gelangen; Afrikaner stranden auf dem Weg nach Westeuropa in Russland; zehntausende Südamerikaner gelangen auf der Suche nach einem besseren Leben nach Spanien, um von dort wiederum nach ganz Europa weiterzuwandern. Während regionale Partnerschaften - wie oben festgestellt - zweifellos notwendig und sinnvoll sind, machen ebensolche Prozesse einen auf globale Partnerschaft ausgerichteten Migrationsdialog unerlässlich.

Mit der Entwicklung globaler Partnerschaften sind jedoch mehrere Herausforderungen verbunden. Zunächst gilt es festzuhalten, dass die Welt in Bezug auf Migration „auf dem Kopf“ zu stehen scheint: Der Norden - normalerweise vehementer Verfechter der Menschenrechte und Befürworter einer globalisierten Welt - verweist mit Bezug zu Fragen der Einwanderung meist nachdrücklich auf die staatliche Souveränität. Der Süden seinerseits betont die Rechte der Migranten und fordert den freien Personenverkehr globalen Massstabs.

Die Diskussionen in Sachen Arbeitsmarkt und Personenfreizügigkeit sind exemplarisch für die vorherrschende Dichotomie zwischen Nord und Süd. Legale Migration, insbesondere wenn sie mit Arbeit verbunden ist, wird vermehrt von Experten aus verschiedenen Perspektiven - unter anderem als Beitrag zur Entwicklung des Herkunftslandes oder als Mittel zur Prävention irregulärer Migration - als positiv betrachtet. Gleichzeitig gibt es namentlich bei Industriestaaten Bedenken, dass eine Konkurrenzsituation für lokale Arbeitnehmer kreiert wird, und dass auch Integrationsschwierigkeiten bestehen könnten, was wiederum zu einem protektionistischen Verhalten bezüglich des Arbeitsmarktes führen kann. Interessant zu verfolgen ist diese fortlaufende Abwägung verschiedener Interessen und Bedenken zum Beispiel im Rahmen der Diskussionen zum so genannten „Mode 4“ des GATS-Abkommens. Dieser Teil des WTO-Abkommens über den Handel mit Dienstleistungen (GATS) definiert den vorübergehenden Grenzübertritt von natürlichen Personen zum Zweck einer Dienstleistungserbringung.

Mit Blick auf diese Positionierung erscheint ein gänzlich neues Migrationsregime, das auf einer neuen internationalen Konvention oder einer neuen UN-Organisation für Migrationsfragen beruhen würde, in absehbarer Zukunft utopisch. 
Was derzeit benötigt wird, ist eine globale Plattform mit der erforderlichen rechtlichen und politischen Legitimation, um im partnerschaftlichen Dialog eine gemeinsame politische Agenda mit Bezug zu den zahlreichen Chancen und Herausforderungen des Migrationsphänomens $\mathrm{zu}$ formulieren. Während bereits mehrere solcher Migrationsforen effektiv existieren, wird längerfristig nur die UNO die nötigen Voraussetzungen erfüllen, um diesen Prozess nachhaltig und inklusiv voranzutreiben. Die erste Zusammenkunft im Rahmen des UN Highlevel Dialogue on International Migration and Development (UN-HLD) im Jahr 2006 vermochte denn auch das Thema „Migration“ erfolgreich auf der internationalen Agenda zu verankern. Gleichzeitig löste der Dialog auf unterschiedlichen Ebenen eine Vielzahl an Prozessen aus, die bis heute dazu beitragen, den internationalen Migrationsdialog qualitativ zu vertiefen.

\section{Der erste UN High-level Dialogue: Werdegang und Perspektiven}

Erste Debatten zum Thema „Migration und Entwicklung“ auf internationaler Ebene fanden 1994 an der Internationalen Konferenz über Bevölkerung und Entwicklung in Kairo statt. Eigentliches Momentum gewann das Thema jedoch erst in den letzten Jahren. Die Schweiz hat mit der Berner Initiative, mit der Unterstützung der Weltkommission für Internationale Migration (GCIM) sowie mit der Lancierung einer Ad-hoc-Staatengruppe in Genf zur Diskussion über Migrationsfragen wesentlich zu der inhaltlichen Vorbereitung des UN-HLD beigetragen.

\section{Die Berner Initiative}

In Anbetracht der Notwendigkeit eines koordinierten Vorgehens zwischen Herkunfts-, Transit- und Zielländern lancierte das damalige Bundesamt für Flüchtlinge - heute Bundesamt für Migration - im Juni 2001 die Berner Initiative. Durch den Aufbau eines Dialogprozesses zwischen den betroffenen Staaten ist es der Berner Initiative gelungen, eine von Regierungen sämtlicher Regionen mitgetragene gemeinsame Orientierung im Migrationsbereich zu definieren, die auf den Grundsätzen von Zusammenarbeit, Transparenz, Vorhersehbarkeit und Ausgewogenheit beruht.

Im Juli 2003 konnte im Rahmen der Berner Initiative eine internationale Konsultationsrunde in Bern organisiert werden, an der ein erster Entwurf einer „Internationalen Agenda für Migrationsmanagement“ (IAMM) ${ }^{5}$ diskutiert wurde. Die IAMM, ein Referenzsystem für das Migrationsmanagement, beruht auf gemeinsamen, von Staaten getragenen Verständnissen (,„common understandings") und erfasst wirksame Praktiken (,effective practices“) im Umgang mit Migration. Sie orientiert sich an internationalen Rechtsnormen und Prinzipien, die im Bereich der Migration Geltung beanspruchen, stellt aber keine rechtlich bindenden Regelungen auf. Der grosse Erfolg der Berner Initiative hat im Jahr 2004 zudem die Durchführung von regionalen Konsultationen in Afrika, Amerika, Asien und Europa ermöglicht. Diese Konsultationen, welche das weltweite

5 International Agenda for Migration Management (IAMM), <http://www.bfm.admin.ch/bfm/de/home/ themen/internationales/multilaterales/international_agenda.html>. 
Interesse an der Berner Initiative förderten, stellten sicher, dass die Anliegen und Perspektiven aller Regionen in der IAMM aufgenommen wurden. Höhepunkt der Berner Initiative bildete die Konferenz „Berne II“, die am 16. und 17. Dezember 2004 erfolgreich in Bern durchgeführt wurde. An dieser Konferenz wurde die endgültige Fassung der IAMM von den rund 300 Regierungsvertretern aus über 100 Staaten sowie von Vertretern internationaler Organisationen als wichtiges und hilfreiches Arbeitsinstrument für das Migrationsmanagement auf nationaler, regionaler und globaler Ebene empfohlen. Mit der Verabschiedung der IAMM endete auch das Mandat der Berner Initiative. Die Agenda bleibt aber weiterhin ein Referenzdokument im Bereich des Migrationsmanagements. Seither wurden in verschiedenen Herkunfts- und Transitregionen mehrere Workshops über die Umsetzung der Empfehlungen und die Verbreitung ihrer Grundsätze durchgeführt.

\section{Global Commission on International Migration}

Der frühere UN-Generalsekretär Kofi Annan bezeichnete die Migration als neue Priorität für die internationale Staatengemeinschaft ${ }^{6}$. Laut einer UN-Arbeitsgruppe über Migration gibt es auf internationaler Ebene im Migrationsbereich normative und institutionelle Lücken und es fehlt an politischer Führungsverantwortung (Doyle Report) ${ }^{7}$. Daraufhin wurde am 9. Dezember 2003, in Anwesenheit von Bundesrätin Micheline Calmy-Rey und der ehemaligen schwedischen Migrationsministerin Holmberg, die Weltkommission für Internationale Migration (Global Commission on International Migration, GCIM) ins Leben gerufen. Die Kommission verfolgte drei Hauptziele: 1. Hervorhebung der positiven Aspekte der Migration; 2. Verdeutlichung der Lücken innerhalb der internationalen Migrationsdebatte; 3 . Formulierung konkreter Handlungsanweisungen.

Am 5. Oktober 2005 wurde der Bericht der GCIM veröffentlicht und dem UNGeneralsekretär unterbreitet. Der Bericht versteht sich als umfassender Rahmen für eine Migrationssteuerung der Staaten auf internationaler, nationaler und regionaler Ebene. Er beruht auf sechs Handlungsprinzipien mit entsprechenden Empfehlungen, insbesondere zu Fragen des Arbeitsmarktes, der Entwicklungszusammenarbeit, der Regelung irregulärer Migration, des Verhältnisses von Gesellschaft und Migration sowie zur Thematik des rechtlichen und normativen Regelwerks der internationalen Migrationsgouvernanz. Der Bericht gilt derzeit als eines der wichtigsten Referenzdokumente für den internationalen Migrationsdialog.

\section{UN High-level Dialogue on International Migration and Development (UN-HLD)}

Mit dem UN-HLD wurde im September 2006 in New York zum ersten Mal auf hohem Niveau über Migration und insbesondere über den Zusammenhang

6 United Nations General Assembly, Strenghtening of the United Nations: An Agenda for Further Change, Report of the Secretary-General, UN-Document A/57/387, New York, United Nations, 9 September 2002, <http://www.un.org>.

7 Michael Doyle, Report to the Secretary General on Migration, New York, 2003, <http://www.un. org >. 
zwischen Migration und Entwicklung im Rahmen der Vereinten Nationen diskutiert. Dabei zeichneten sich die folgenden Diskussionsschwerpunkte ab:

$\square$ Zwischen Migration und Entwicklung besteht ein enger Zusammenhang. Einerseits können Entwicklungsprogramme im Bereich der Armutsbekämpfung und der Gouvernanz, aber auch Massnahmen im Bereich der Friedensförderung und der Menschenrechte zur Verringerung der aus Not erfolgenden oder erzwungenen Migration beitragen. Andererseits leisten Migranten mit ihren Geldüberweisungen, aber auch durch den Transfer von Wissen, Idealen und Modellen (z.B. Demokratie, gewaltfreies Zusammenleben) einen wichtigen Beitrag zur Entwicklung ihrer Heimat.

- Migration ist untrennbar mit Arbeit verbunden. Die Aussicht auf Arbeit ist einer der Hauptgründe sowohl für die reguläre, als auch für die irreguläre Migration. Die Schaffung von Arbeitsplätzen in den Entwicklungsländern selbst ist daher ein gutes Mittel, um unerwünschte und irreguläre Arbeitsmigration zu bekämpfen. Gleichzeitig bleibt die Forderung nach Erweiterung der Möglichkeiten legaler Arbeitsmigration - kontrollierte Öffnung des Arbeitsmarktes, vereinfachte Anerkennung von Diplomen, Einrichtung zirkulärer Migrationsformen - eine Konstante in den Stellungnahmen der Entwicklungsländer. Dies kann in diesen Ländern allerdings auch zu einem Mangel an dringend benötigten, qualifizierten Arbeitskräften führen.

- Negative Aspekte der Migration müssen bekämpft und die Rechte der Migranten geschützt werden. Menschenhandel, Menschenschmuggel und alle Formen der Ausbeutung müssen bekämpft werden. Die Opfer sollen geschützt und die Täter verfolgt werden. Durch geeignete Partizipations- und Integrationsmassnahmen können und müssen Diskriminierung und Xenophobie in den Aufnahmeländern verringert werden. Zudem muss die negative Wahrnehmung der Migration in der Gesellschaft durch mehr praxisorientierte Forschung und Erkenntnisse über die verschiedenen Aspekte der Migration relativiert werden. Die Einhaltung der Menschenrechte und die Berücksichtigung von Gender-Aspekten in der Politikentwicklung sollen zu grundlegenden Prinzipien in der internationalen Migrationsdebatte werden.

\Zusammenarbeit und Partnerschaften müssen gefördert werden: Regierungen von Herkunfts-, Transit- und Zielländern, welche die Hauptverantwortlichen für ein gutes Migrationsmanagement sind, sollen ihre Zusammenarbeit vermehrt durch multi- und bilaterale Vereinbarungen stärken. Partnerschaften müssen aber auch mit dem Privatsektor, der Zivilgesellschaft und mit internationalen Organisationen angestrebt werden.

Die UN-Vollversammlung hat in einer Resolution vom Dezember 2006 ausdrücklich das erhöhte Bewusstsein im Umgang mit der Migrationsthematik begrüsst und gleichzeitig beschlossen, anlässlich der 63. Generalversammlung mögliche Optionen für angemessene Folgeaktivitäten zu prüfen ${ }^{8}$. Die Schweiz setzt sich dafür ein, regelmässige Treffen des hochrangigen Dialogs (High-level Dialogue) im Rahmen der UN-Generalversammlung alle drei bis vier Jahre durchzuführen. Dies würde eine willkommene Verankerung der Migrations-

8 United Nations General Assembly, International Migration and Development, Resolution adopted by the General Assembly, UN-Document A/RES/61/208, New York, United Nations, 6 March 2007, $<$ http://www.un.org $>$. 
thematik innerhalb der UNO begünstigen und durch eine periodische Bestandsaufnahme von Aktivitäten sicherstellen, dass das Thema „Migration“ weiterhin auf der internationalen Agenda bleibt.

\section{Global Forum on Migration and Development (GFMD)}

Die Teilnehmerstaaten waren sich mehrheitlich einig, dass der hochrangige Dialog über Migration und Entwicklung (UN-HLD) der Grundstein für eine weiterführende internationale Migrationsdebatte sein sollte. Eine Gruppe von Staaten ${ }^{9}$ schlug deshalb vor, ein informelles Forum über Migration und Entwicklung zu errichten. Das Weltforum für Migration und Entwicklung (Global Forum on Migration and Development, GFMD) ${ }^{10}$ soll der Staatengemeinschaft die Möglichkeit bieten, dieses komplexe Thema lösungsorientiert anzugehen, ohne dabei über normative Grundsätze zu diskutieren. Die erste Konferenz des Forums fand im Juli 2007 in Belgien statt. Weitere Konferenzen sind auf den Philippinen sowie in Griechenland, Argentinien, Spanien und Marokko vorgesehen.

Das Forum muss die Dynamik im Migrationsbereich nutzen, die dank des UNHLD entstanden ist, und sollte zu einer informellen Plattform für Staaten werden, um den Dialog im Migrationsbereich weiterzuführen. Dadurch können gemeinsame Ziele formuliert und deren Erreichung vorangetrieben werden. Die Diskussionen sollen zu konkreten bi- und multilateralen Projekten führen, die im Nachgang zum Forum verwirklicht und umgesetzt werden. In diesem Sinne wird das Forum idealerweise zu einem Katalysator für Migrationsprojekte zwischen Staaten, bzw. zwischen Staaten und weiteren Akteuren, wie internationale Organisationen und/oder Nichtregierungsorganisationen. Die ersten Erfahrungen aus Brüssel sind ermutigend und deuten an, dass diese Plattform die hoch gesteckten Ziele erfüllen kann. Es gilt indes zu betonen, dass das Forum niemals jenes politische Gewicht erhalten wird, das dem UN-HLD zukommt. Das Weltforum für Internationale Migration und Entwicklung (GFMD) ist damit weder als ein formeller Folgeprozess des UN-HLD zu verstehen, noch kann es diesen ersetzen.

\section{Ausblick}

Die schweizerische Migrationspolitik besitzt eine Reihe von zukunftsgerichteten Instrumenten, um das Potenzial des Nexus zwischen Migration und Entwicklung im Sinne aller beteiligten Partner umsetzen zu können. Trotz der positiven Erfahrungen auf dem Gebiet der Rückkehrhilfe und der engen Verknüpfung der Politikbereiche Migration und Entwicklung innerhalb der zukünftigen Migrationspartnerschaften kann auch die Schweiz die inhärenten Synergien im Bereich Migration und Entwicklung noch vermehrt nutzen. Durch eine aktive Teilnahme an den regionalen und internationalen Initiativen wird die Schweiz nicht nur ihre Erfahrungen einbringen können, sondern auch aus den Ideen und Erkenntnissen anderer Staaten Nutzen ziehen können. Das EDA -

9 Zu dieser Gruppe gehörten Belgien, Brasilien, Kanada, Marokko, Mexiko, die Niederlande, Nigeria, die Philippinen, Spanien und Schweden.

10 Siehe <http://www.gfmd2008.org>. 
insbesondere die Politische Abteilung IV Menschliche Entwicklung - versteht sich diesbezüglich auch als Scharnierstelle zwischen Ausland und Inland. Sie macht es sich zur Aufgabe, die migrationspolitischen Interessen der Schweiz im Ausland zu vertreten und gleichzeitig die Aussenperspektiven in den internen Diskurs einfliessen zu lassen und, wo sinnvoll und angemessen, auch in die Politikentwicklung mit einzubeziehen.

Wir sehen uns allerdings auch vor neue Herausforderungen gestellt. Denken wir an den Klimawandel, der unweigerlich einen Einfluss - ob direkt oder indirekt auf die globalen Migrationsströme haben wird. Oder an die zunehmend gemischten Wanderbewegungen (sog. ,mixed migratory flows“"), wo Flüchtlinge und Migranten in die gleichen Menschenströme gelangen und die Staaten vor die Schwierigkeit stellen, den angemessenen Status der einzelnen Personen zu definieren. Die PA IV wird diese verschiedenen Entwicklungen zusammen mit dem federführenden Bundesamt für Migration (BFM) und weiteren Stellen der Bundesverwaltung genau verfolgen und angemessene Antworten im Sinne unserer Migrationspolitik und des Schutzes anfälliger Personen formulieren.

Schliesslich sollten wir vermehrt Umsetzungsmöglichkeiten, auch unter Einbezug der Zivilgesellschaft, der Forschung, des Privatsektors, der Nichtregierungsorganisationen und nicht zuletzt der lokalen Diaspora diskutieren. Die genannten Organisationen können wichtige Partner für eine breit abgestützte Migrationspolitik sein, die vermehrt auch die Synergie mit der Entwicklungspolitik sucht und die Bevölkerung in einen fortwährenden Dialog über die Migration einbezieht. Denn der Handlungsspielraum einer nationalen Migrationspolitik wird auch durch die Wahrnehmung dieser Politik in der Gesellschaft mit beeinflusst. Solange Migranten eher als eine Gefahr - beispieslweise für die soziale Kohäsion oder für den eigenen Arbeitsplatz - und nicht als Potenzial für die soziale und wirtschaftliche Entwicklung des Landes angesehen werden, sind die Gestaltungs- und Umsetzungsmöglichkeiten einer offenen und innovativen Migrationspolitik begrenzt. Die PA IV wird auch in Zukunft einen Beitrag für diesen offenen Dialog mit der Gesellschaft leisten, so wie sie es unter anderem mit ihrer Jahreskonferenz 2007 zum Thema „Migration: Chancen und Herausforderungen"11 getan hat.

11 Siehe <http://www.eda.admin.ch/eda/de/home/dfa/orgcha/sectio/pad/pad4.html>. 